\title{
Jahres-Inhalt 1991/Complete Contents 1991
}

\section{Editorial}

Die Infusionstherapie

Reissigl, $\mathrm{H}$

Übersichtsarbeiten

L-Tryptophan und das Eosinophilie-Myalgie-Syndrom - Konsequenzen für die künstliche

Ernährung?

Bäßler,K.H

Anaesthesiologie und Transfusionsmedizin*

Bergmann, $\mathrm{H}$

Originalarb $\theta$ iten

Wie zuverlässig sind Kurzzeitmessungen

der Sauerstoffaufnahme bei polytraumatisierten und langzeit-beatmeten Patienten?**

Behrendt,W., Surmann, M., Raumanns, J., Giani, G

\section{2rbeiten}

Lipolyse und Lipidoxidation bei gewichtsstabilen Patienten mit malignen Tumoren des

Verdauungstraktes

Selberg, O., Weimann, A., Meyer, H.J., Canzler, H.,

Müller, M.J

Kurzdauernde Infusionstherapie im Kindesalter. Ein Vergleich individuell gemischter mit kommerziell erhältlichen Infusionslösungen

Fusch, C, Moeller, H

Schwankungen der Serumkonzentrationen von Anti-A und Anti-B Histo-Blutgruppen-

Antikörpern bei einer Patientin nach Lebertransplantation*

Zimmerli, M., Lerut, J., Müller, P., Zimmermann, A.,

Nydegger, U

Abnahme der Konzentrationen von Tobramycin, Vancomycin und Phenobarbital bei Gabe über Infusionsfílter

Böhrer, H., Zhang, C.H., Krier, C

85

91

Kontrolle der postoperativen enteralen Ernährung mit dem H2-Atemtest

Kemen, M., Homann, H.-H., von Liebe, S., Zumtobel, V.

Gesellschaftsmitteilungen

Mitteilungen der Deutschen Gesellschaft für Trans

fusionsmedizin und Immunhämatologie (DGTI)

Kurzmitteilungen 
Herstellung leukozytenarmer Thrombozyten-konzentrate: In-vitro-Testung eines neuen Filtersystems (PL 100)

Böck, M., Himmelsbach, S., Gudden, A., Greither, L.,

Mempel, W

SO

Originalarbeiten

Untersuchungen zum postoperativen Stickstoffverlust

Müller, J.M., Jerke, A.S., Wolters, U

Infusionsmischungen - Untersuchungen der Praxis in Osterreich

Haslinger-Matzenauer, M., Karger, H., Kirchdorfer, K., Petcold, L., Rosmann van Goethem, J., Kerbl, H., Zadravec, S.

109

114

Gesellschaftsmitteilungen

Mitteilungen der Deutschen Gesellschaft für Transfusionsmedizin und Immunhämatologie (DGTI). .

55

Journal Reviews $\quad 36$

Abstracts

Abstracts zur 10. Gemeinsamen Jahrestagung der

Deutschen Arbeitsgemeinschaft für künstliche

Ernährung (DAKE) und der Österreichischen

Arbeitsgemeinschaft für klinische Ernährung (AKE),

20.-23. März 1991, Göttingen 47

Beeinflußt variierte parenterale Zinkzufuhr die Interaktion zwischen Tumor und Wirt?

Untersuchungen anhand eines Tiermodells

Wolters, U, Müller, J.M., Wölfelschneider, K., Inland, H 123

Eine neue Methode zur Bestimmung des zirkulierenden Blutvolumens Erythrozytenmarkierung mit Fluorescein-Natrium

Lauermann, 1130

Kurzmitteilungen

Lagerung von Thrombozytenkonzentraten: Ultrastrukturelle und funktionelle Veränderungen

Böck, M., Greither, L., Gudden, A., Diehm, H., Heim, M.U.,

Mempel. W 137

Jahres-Inhalt 1991/Complete Contents 1991

317

Tumorzellseparation durch Cell-Saver und Membranfilterpassage

Wiesel, M., Güdemann, C, Staehler, G

143

Journal Reviews $\quad 146$

Gesellschaftsmitteilung

Mitteilung der Deutschen Gesellschaft für Transfusionsmedizin und Immunhämatologie (DGTI). .150

Übersichtsarbeiten 
Kohlenhydrate in parenteralen Nährlösungen für die Pädiatrie - eine kritische Bewertung Heine, W

160

Origínalarbeiten

Parenteral verabreichte mittelkettige Triglyzeride induzieren Veränderungen im Carnitin-

Stoffwechsel

Rössle, C, Carpentier, Y.A., Richelle, M., Dahlan, W.11/8

D'Attellis, N.P.D., Elwyn, D.H., Stehle, P., Fürst, P 167

Applikations- und Fixationssysteme der enteralen Ernährung

Rabast, U 172

Zugangswege der parenteralen Ernährung

Löhlein, D 182

Vergleich verschiedener Thrombozytapherese-Verfahren*

Kretschmer, V 188

Originalarbeiten

Der Einfhili einer L-Carnitin-Supplementierung auf den Lipidstoffwechsel niereninsuffizienter, dialysepflichtiger Kinder und Jugendlicher

Böhles, H., Michalk, D., v.Wendt-Göknur, E224

Freie Fettsäuren als Indikator für präoperativen Streß sowie der Einfluß einer Prämediktion mit Flunitrazepam, Morphium und Promethazin auf den Fettsäurespiegel im Plasma

Hofmann, M., Kleemann, P.P227

Postoperative Dünndarmmotilität nach abdominalchirurgîschen Eingriffen

Kemen, M., Bein, N., Homann, H.H., Bauer, K.H.,

Zumtobel, V 233

Die Speicherung von zwei unterschiedlichen Hydroxyäthylstärke-Präparaten in der Plazenta nach Hämodilution bei Patientinnen mit fetaler Mangelentwicklung oder

Schwangerschaftshochdruck

Heilmann, L., Lorch, E., Hojnacki, B., Müntefering, H.,

Förster, H 236

Thrombozytapherese mit dem Zellseparator A 201 (Baxter) - erste Daten zur Biokompatibilität Moog, R., Luboldt, W, Krützfeldt, A., Paar, D.,

Holtmann, M., Mengelkamp, A.K 244

Massiv- und Multitransfusion bei polytraumatisierten Patienten: Langfristige serologische

Befunde zu Hepatitis B, Hepatitis C und AIDS

Schneck, H.J., Dobler, G., v. Hundelshausen, B.,

Nathrath, M., Drescher, M 248

Briefe an den Herausgeber 256

Kongreßbericht 259

Kurzmitteilungen

Transfusionsergebnisse mit Plättchenkonzentraten von zwei verschiedenen Zellseparatoren*

Kretschmer, V., Planitz, C, Dietrich, G., Pflüger, H.H. . .

Der Thrombin-Antithrombin-III-Komplex-ELISA-Test in der Diagnostik der

operationsbedingten Gerinnungsaktivierung

Kemen, M., Sperling, H., Mumme, A., Zumtobel, V. . . .

196 
199Übersichtsarbeiten

Posttraumatische hypokalorische parenterale Ernährung -Entwicklung und klinische

Anwendung*

Behrendt, W., Surmann, M 271

Sind $\Omega$-3-Fettsäuren für das Neugeborene essentiell?

Lothaller, M.-A., Widhalm, K 280

Computerprogramm PEDINFUS für die totale parenterale Ernährung von Kindern

Kuchenbecker, J 285

Übersichtsarbeiten

Ätiologie, Pathophysiologie und klinische Bedeutung der hereditären Fruktoseintoleranz

Fauth, U., Halmágyi, M

213Originalarbeiten

Radiologische und klinische Kontrolluntersuchung zentraler Venenverweilkatheter bei heimparenteraler Ernährung (HPN)

Hofmann-Preiß, K., Becker, A., Sailer, S Jahres-Inhalt 1991/Complete Contents 1991

Die kurzfristige Wirkung des Argininhydrochlorids auf die $\Lambda$ minosäurenkonzentrationen im Serum der Ratte

Doehn, M., Schulze, C.G 297

Auswirkungen einer definierten Infusion von 10\% HAES 200/0,5 in der Frühphase eines

septischen Syndroms auf die hämodynamischen Parameter

Simon, H.B., Mertens, H., Löhmer, P., Agternkamp, C,

Klosterhalfen, B., Müller, F.G 301

Untersuchungen zur Verträglichkeit von Haemofusin in der Hämodilution und

Volumensubstitution

Kaniecki, K., Biesel, E., Zielke, E

306

Gesellschaftsmitteilung

Mitteilung der Deutschen Gesellschaft für Transfusionsmedizin und Immunhämatologie (DGTI) .. 311

Spielt die Leukozyten-Depletion eine wichtige Rolle bei der Vermeidung von

Alloimmunisierung durch Einzelspender-Thrombozytentransfusionen?

Gmür, J., Schanz, U., Burger, J., Reichlin, M.·, Müller, E.,

Oelz, 013

Filtration von Thrombozytenpräparaten

Müller, N 19

Thrombozytapherese mit der neuen COBE Spectra ${ }^{\circledR}$

Kretschmer, V., Rossa, W., Eisenhardt, G 24

Das Leistungspaket des COBE Spectra ${ }^{\circledR}$-Apherese-Systems

Uhrdahl, S.G 29

Zukünftige Technologien in der Transfusionsmedizin

Klein, H.G 31

*Diese Arbeit erscheint in englischer Sprache

Supplement 1 
Methoden zur Plättchenlagerung

Murphy, S

Supplement 2

Abstracts des gemeinsamen Kongresses der European Society for Haemapheresis (ESFH) und der Deutschen Gesellschaft für Transfusionsmedizin und Immunhämatologie (DGTI) in Würzburg, 8.-14. September 1991

Auswirkungen der Leukozyten auf die Lagerung von Thromboytenkonzentraten

Peitersz, R.N.I., de Korte, D., Dekker, W.J.A., Reesink, H.W. . . 10 